\title{
EFECTO DE LA PRÁCTICA MENTAL Y FÍSICA EN EL DESEMPEÑO MOTOR Y ACTIVIDAD ELÉCTRICA MUSCULAR EN ESTUDIANTES SANOS
}

\section{THE EFFECT OF MENTAL AND PHYSICAL PRACTICE IN MOTOR PERFORMANCE AND MUSCLE ELECTRICAL ACTIVITY IN HEALTHY STUDENTS}

\author{
Alfredo Urcuyo Ovares ${ }^{1}$, Jefferson Ávila Chaverri ${ }^{1}$, Judith Jiménez Díaz ${ }^{1,2}$ y Bryan Montero \\ Herrera $^{1}$ \\ ${ }^{1}$ Escuela de Educación Física y Deportes, Universidad de Costa Rica, Costa Rica \\ ${ }^{2}$ Centro de Investigación en Ciencias del Movimeinto Humano, Universidad de Costa Rica, Costa \\ Rica
}

\begin{abstract}
alfredourcuyo@gmail.com; jeffavilach@gmail.com; judith.jimenez d@ucr.ac.cr; bryan mh2005@hotmail.com
\end{abstract}

Envío original: 2020-01-30 Reenviado: 2020-05-11 Aceptado: 2020-06-01

Publicado: 2020-06-11

Doi: https://doi.org/10.15517/pensarmov.v18i1.40368

\begin{abstract}
RESUMEN
El objetivo del presente estudio fue analizar la efectividad de la práctica mental (PM), práctica física (PF) y su combinación (PC) en el desempeño de la destreza de dribbling de hockey de salón y la actividad eléctrica del músculo (AEM) braquiorradial del antebrazo dominante. Un total de 27 estudiantes universitarios de primer año de carrera en Ciencias del Movimiento Humano, sin experiencia previa en la destreza, fueron asignados aleatoriamente a uno de tres grupos (PF, PM y PC). El desempeño de la destreza fue evaluado por medio del tiempo total utilizando fotoceldas SmartspeedPro y la AEM se midió con un electromiograma Noraxon M400. La investigación se desarrolló en dos sesiones. En la primera sesión se realizó el pretest, la práctica experimental según el grupo correspondiente y el posttest del desempeño y AEM. En la segunda sesión, una semana después, se evaluó el desempeño en una prueba de retención. Al aplicar un análisis de varianza (ANOVA) de 2 vías mixto [grupo (3) x medición (3)] se encontró que el desempeño de los tres grupos mejoró significativamente en la prueba de adquisición y retención. EI ANOVA de 2 vías mixto [grupo (3) x medición (2)] indicó que no hubo diferencias significativas en la AEM. La PF, PM y PC favorecieron el aprendizaje de la destreza, sin embargo, no se encontró cambios en la actividad eléctrica del músculo braquiorradial del antebrazo dominante.
\end{abstract}


Palabras clave: aprendizaje motor, adquisición, retención, hockey de salón.

\begin{abstract}
The purpose of this study was to analyze the effect of mental practice (MP), physical practice (PP) and its combination (CP), in the performance of a dribbling skill in field hockey, and muscle electrical activity (EMA) in the brachioradialis of the dominant forearm. A total of 27 college students in the Human Movement Science career, without experience in the skill, were randomly assigned into one of the three groups (MP, PP, and CP). The skill performance was assessed through total time using SmartspeedPro photocells and MEA was tested by a Noraxon M400 electromyogram. The research had two sessions. In the first session the pretest for motor performance and EMA was assessed, participants were randomly assigned to one of three groups, and after the practice, the posttest was assessed. One week later, in the second session a retention test for motor performance was assessed. A two-way mixed ANOVA [group (3) x measurement (3)] found significant improvements for performance in the three groups for acquisition and retention test. The two-way mixed ANOVA [group (3) $x$ measurement (2)] pointed out no significant differences in MEA. The PP, MP, and CP enhances skill learning, nevertheless, no significant changes were founded in the brachioradialis muscle electrical activity of the dominant forearm.
\end{abstract}

Keywords: motor learning, acquisition, retention, field hockey.

La práctica mental (PM), también conocida como entrenamiento de habilidades mentales, imaginería motora, o preparación mental es la representación de una acción motriz específica a nivel mental, pero con la particularidad de que no hay ninguna respuesta motora visible (Frenkel et al., 2014; Liu, Song y Zhang, 2014; Olusoga, Maynard, Butt y Hays, 2014; Vodičar, Kovač y Tušak, 2012). Comúnmente, la PM se clasifica en PM visual (PMV) y PM kinestésica (PMK) (Frenkel et al., 2014). La PMV se caracteriza porque la acción, objeto o escena se imagina en tercera persona, es decir, imaginando a alguien más realizado la acción. La segunda clasificación se diferencia de la visual desde una perspectiva de primera persona, por lo tanto, se le pide a quien la ejecuta sentir y/o experimentar las sensaciones propias del movimiento que están haciendo (Frenkel et al., 2014). Por ejemplo, cuando una persona se imagina levantando una mancuerna de un determinado peso, en la PMK debe tratar de percibir — sin moverse— las 
contracciones musculares, las sensaciones de fatiga y la fuerza que se ejerce durante dicho movimiento, mientras que, en la PMV solo se imaginan a una persona realizando la destreza. Se ha detectado que la PMK, presenta mayores beneficios en comparación con la PMV (Frenkel et al., 2014; Zapala et al., 2014), ya que activa las regiones cerebrales asociadas con la corteza motora, mientras que la PMV activa las relacionadas con la corteza visual (Guillot et al., 2009; Stecklow, Infantosi y Cagy, 2010). Cabe destacar que, ambas cortezas (motora y visual) están involucradas en el proceso de preparación y anticipación, durante la primera fase de aprendizaje del movimiento (Jackson, Lafleur, Malouin, Richards y Doyon, 2003).

La PM ha sido estudiada como método de entrenamiento, en el área del movimiento humano, para las capacidades físicas (Rozand, Lebon, Papaxanthis y Lepers, 2014), para la mejora del desempeño y aprendizaje de destrezas motoras (Azimkhani, Abbasian, Ashkani y Gürsoy, 2013; Feltz y Landers, 1983; Gomes et al., 2014; Wriessnegger, Steyrl, Koschutnig y Müller-Putz, 2014) e incluso en psicología deportiva, en el manejo de ansiedad precompetitiva (Vodičar et al., 2012), presentado mejorías significativas.

En el área de aprendizaje motor, se ha aplicado la práctica mental, la práctica física (PF) y su combinación (práctica combinada; PC), tanto en pruebas de adquisición, como en pruebas de retención o transferencia, con el objetivo de determinar su efecto en el aprendizaje de destrezas. Al comparar la efectividad entre PM y no realizar ningún tipo de práctica en un grupo de deportistas novatos (edad 19-20 años) encontraron una mejora en el desempeño en el golpe de derecha y revés en tenis y en el pase de hockey, después de 16 sesiones (en seis semanas) de realizar PM, no así para el grupo sin práctica (Hegazy, Sherif y Houta, 2015). Por otro lado, al comparar diferentes tipos de práctica: PF, PM y PC en el desempeño de una destreza de balonmano se encontró que en la etapa de adquisición el grupo que realizó PC presentó un mejor desempeño en comparación con los grupos de PF, PM o no realizar práctica, después de una práctica de 20 intentos; mientras que en la prueba de retención el grupo que realizó PF presentó un desempeño similar al grupo de PC, y ambos grupos presentaron un mejor desempeño que el grupo de PM; además realizar PM es mejor que no realizar ningún tipo de práctica (Azimkhani et al., 2013). Adicional a lo anterior, se encontró que realizar PF y PC presenta un mejor resultado en el desempeño que realizar solo PM o no realizar ningún tipo de práctica, al ejecutar una destreza de precisión en serie en un grupo de estudiantes universitarios, en este caso la duración de la práctica fue de 6 intentos (Gomes et al., 2014). Además, por el contrario, en un estudio se encontró que al realizar PC durante 8 sesiones (ya sea 8 minutos o 12 minutos por sesión) favorece el desempeño en una kata en comparación con realizar práctica física de la kata (Navarro, Araya-Vargas y Salazar, 2002). Los beneficios de la PM se han presentado tanto en 
sesiones de un día (Azimkhani et al., 2013; Gomes et al., 2014), con diferentes cantidades de intentos, como en sesiones de dos días o más (Hegazy et al., 2015; Navarro et al., 2002). Feltz y Landers (1983) determinaron que la cantidad de intentos explican el $20 \%$ del tamaño de efecto al realizar PM.

La evidencia ha mostrado que la PM favorece un mejor desempeño, cuando se compara con no realizar ningún tipo de práctica (Feltz y Landers, 1983; Gomes et al., 2014; Hegazy et al., 2015). Pero, se ha demostrado que la PF es superior que la PM (Azimkhani et al., 2013; Feltz y Landers, 1983; Gomes et al., 2014). A su vez se ha identificado que la PC favorece aún más el desempeño motor en comparación con realizar sólo PF o PM (Azimkhani et al., 2013; Navarro et al., 2002). Este beneficio se ha visto luego de prácticas de una sola sesión con pocos intentos (Azimkhani et al., 2013; Gomes et al., 2014), como en estudios con intervenciones de práctica de más de una sesión (Hegazy, et al., 2015; Navarro, et al., 2002). Se sugiere que el beneficio de la PC se debe a la integración sensorial aportados al realizar PF después de haber realizado PM, lo que permite tener un mejor aprendizaje de la destreza, en comparación con realizar cada práctica por separado (Crews y Kamen, 2006). Sin embargo, existe poca evidencia contundente del efecto de la PM para el aprendizaje motor en participantes novatos (Gomes, et al., 2014).

Existen dos hipótesis implicadas en el beneficio que se demuestra al utilizar la práctica mental. La hipótesis de actividad cerebral y la hipótesis de actividad neuromuscular (Magill y Anderson, 2013). Además, se menciona la teoría psiconeuromuscular (Sánchez y Lejeune, 1999). En la primer hipótesis se rescata que la PM es capaz de activar las zonas cerebrales, similar a la PF y esto se ha demostrado en diversos estudios, ya que se ha visto dicha activación, durante ambos tipos de práctica, en el área motora suplementaria, la corteza premotora lateral, los ganglios basales y el cerebelo (Jackson et al., 2003; Lotze et al., 1999; Luft, Skalej, Stefanou, Klose y Voigt, 1998; Matsuda et al., 2011; Miller et al., 2010; Stecklow et al., 2010). En la segunda hipótesis se plantea que, además de la activación en el cerebro, se activan conexiones nerviosas entre el cerebro y el músculo implicado (Magill y Anderson, 2013). De manera similar, la teoría psiconeuromuscular propone que, durante la representación de una actividad, el cerebro envía débiles estimulaciones a través de la médula espinal y luego estas son transferidas a los músculos implicados en la acción representada donde se emitirán pequeñas señales eléctricas (Sánchez y Lejeune, 1999). Estas dos propuestas se respaldan con estudios donde se ha encontrado, por medio de la electromiografía muscular (EMG), activación eléctrica de los músculos durante la práctica mental (Guillot et al., 2007).

El aprendizaje de una nueva destreza podría requerir de prácticas extensas para lograr un desempeño satisfactorio, lo cual podría generar fatiga, aburrimiento, frustración, entre otros, 
(Pangrazi y Beighle, 2019). Sin embargo, se ha demostrado que utilizar métodos complementarios como la PM, favorece en alguna medida el desempeño y aprendizaje de la destreza. Por lo tanto, el objetivo del presente estudio fue analizar la efectividad de la PM, PF y su combinación en el desempeño de la destreza de dribbling de hockey de salón y la actividad eléctrica del músculo braquiorradial del antebrazo dominante, en estudiantes universitarios novatos y sanos. Se plantearon las siguientes hipótesis: 1. los tres grupos de práctica mejoran el desempeño y la actividad eléctrica del músculo (AEM) de la destreza en la adquisición y retención; y 2. tanto en la prueba de adquisición, como de retención, el grupo de PC presentarían mejor resultado (en desempeño y AEM) que el grupo de PF, y ambos presentarían mejor desempeño y AEM que el grupo de PM.

\section{METODOLOGÍA}

Diseño

Se contó con un grupo de estudiantes de Ciencias del Movimiento Humano seleccionados por conveniencia, donde se realizó una asignación aleatoria de los participantes a cada uno de los tres grupos experimentales (práctica física, práctica mental kinestésica, práctica combinada). Se aplicó el "Paradigma de Transferencia" (Shea y Wright, 1997) el cual propone dos fases de evaluación: adquisición y retención; utilizado en variedad de estudios en el área de aprendizaje motor. La asignación aleatoria se realizó tipo tómbola, el nombre los estudiantes se colocaron en papeles separados, y se seleccionó el papel uno a uno, para realizar tres grupos, luego cada grupo fue asignado de manera aleatoria a uno de los tres tratamientos.

\section{Participantes}

Un grupo de 27 estudiantes universitarios voluntarios (edad= $20.96 \pm 4.47$ años) de primer año de la carrera de Ciencias del Movimiento Humano de una Universidad en Costa Rica, físicamente activos y sin lesiones o padecimientos que limitaran su movimiento. No tenían experiencia en la destreza de dribbling con palo de hockey. Se cumplieron los criterios establecidos en la declaración de Helsinki. Se inició el estudio con 30 estudiantes, sin embargo, tres participantes no se presentaron a la medición de retención.

\section{Instrumentos}

Se utilizó un equipo de fotoceldas Smartspeed pro (FCC ID: UQM-SMARTSPEED) para medir el desempeño motor (tiempo en segundos) de la destreza de dribbling con palo de hockey 
en salón. Para realizar el dribbling, se delimitó un espacio de ocho metros de largo por cuatro metros de ancho, en este espacio se dibujó una línea recta de $6 \mathrm{~m}$, se colocaron cinco conos separados por una distancia de $90 \mathrm{~cm}$. En la línea de $6 \mathrm{~m}$, en el metro cero se colocó la primera fotocelda y en el metro seis se colocó la segunda, dejando $1.2 \mathrm{~m}$ entre la primera fotocelda y el primer cono, y entre el último cono y la última fotocelda (figura 1). Se registró el tiempo total de la ejecución de la destreza.

Para medir la actividad eléctrica muscular (AEM) del músculo braquiorradial del antebrazo dominante se usó un equipo de electromiografía marca NORAXON-MYOTRACE 400, junto con el software MyoResearch XP MT400 Clinical Edition 1.07.41.

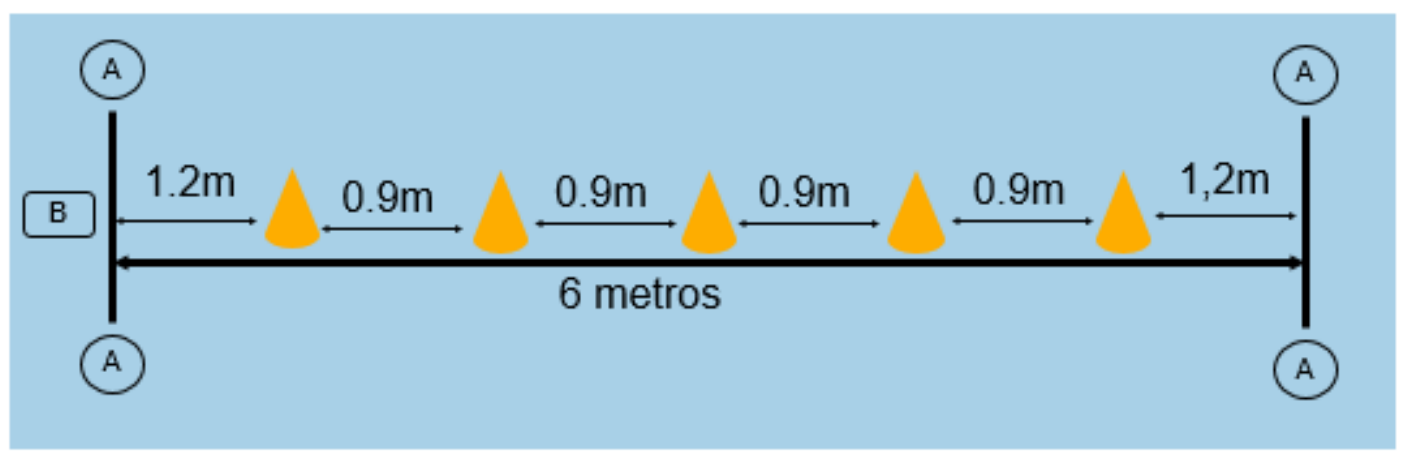

Figura 1. Distribución de los instrumentos y el circuito de dribbling para evaluar el desempeño. Nota: $A=$ posición de las fotoceldas, $B=$ posición de salida de los participantes. Fuente: elaboración propia.

\section{Procedimientos}

El estudio completo consistió en dos sesiones. Una semana antes de iniciar, a las personas interesadas, se les explicó los procedimientos del estudio y se les solicitó firmar el consentimiento informado. En la primera sesión se aplicó el pretest, el tratamiento y la prueba de adquisición, donde se evaluó el desempeño motor (DM) de la destreza y la actividad eléctrica muscular (AEM). En la segunda sesión se realizó la medición de retención, donde solamente se evaluó el DM.

EI DM se midió por medio del tiempo que cada participante duró en realizar el dribbling. Se registró el mejor tiempo de dos intentos. Para la medición de la AEM, se limpió con alcohol los tres puntos donde se colocaron los electrodos a nivel del antebrazo del brazo dominante (figura 2), este paso permite a los electrodos captar mejor la señal y disminuir por consiguiente la impedancia que conlleva a generación de ruido en la señal final (Stegeman y Hermens, 2007). Después, se registró el promedio de tres contracciones voluntarias máximas, a los participantes 
se les solicitó estirar el brazo y cerrar el puño con su mayor fuerza durante dos segundos, y luego relajar. Para registrar el inicio de todas las señales se fijó un nivel basal para todos los participantes. Las señales del EMG fueron limpiadas utilizando las funciones de rectificación - descarta los datos negativos y mantiene solo los positivos (Reaz, Hussain y Mohd-Yasin, 2006) - y suavizado -elimina o reduce la señal que es producida cuando un grupo de motoneuronas disparan al mismo tiempo produciendo una superposición de la señal (Konrad, 2005).

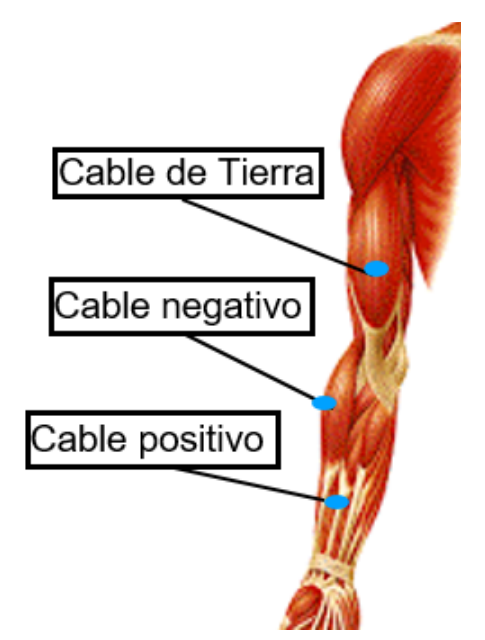

Figura 2. Posición de los electrodos en el brazo para medir la AEM. Fuente: elaboración propia.

Posteriormente, se asignó aleatoriamente a los participantes a uno de tres grupos: práctica mental (PM), práctica física (PF) y combinada (PC). La práctica del grupo de PM consistió en imaginarse realizando el movimiento del dribbling alrededor de los conos, evitando por completo la contracción muscular o el movimiento real, con un enfoque de PMK, debido a que esta práctica prevé más beneficios que la PMV. El participante fue guiado en su entrenamiento mental por medio de un audio que tuvo una duración de 2 minutos y 47 segundos (apéndice 1). El audio se repitió 10 veces, lo que equivale a 10 intentos de la destreza. El lugar utilizado se mantuvo en silencio absoluto y fue un ambiente cómodo para que los participantes no se distrajeran.

El grupo de PF, realizó el dribbling con el palo de hockey ("stick") y la pelota en 10 intentos, con un descanso promedio de 30s entre cada intento. El grupo de PC realizó 5 intentos de PM y 5 intentos de PF, para un total de 10 intentos. Todas las prácticas se llevaron a cabo bajo la supervisión de un investigador y todos los participantes recibieron instrucciones estandarizadas para la realización de las mediciones. Al finalizar la práctica se evaluó la AEM y el desempeño de 
la destreza en una prueba de adquisición. Una semana después, en la segunda sesión, se evaluó el DM en una prueba de retención.

\begin{abstract}
Análisis estadístico
Inicialmente se analizaron los supuestos de normalidad de los datos. Se obtuvo promedios y desviaciones estándar. Se aplicó un análisis de varianza (ANOVA) de dos vías mixto con medidas repetidas en el último factor (3 tratamientos $\times 2$ mediciones), para el análisis de la AEM. Mientras que para el análisis del desempeño motor se aplicó un ANOVA de dos vías mixto con medidas repetidas en el último factor (3 tratamientos $\times 3$ mediciones). De encontrar resultados significativos se aplicaron los análisis post-hoc de Bonferroni. Los análisis fueron realizados con el programa estadístico para las ciencias sociales (SPSS Inc. Chicago, Illinois, USA) versión 24.0. Se consideró estadísticamente significativo un valor $p<0.05$. Además, se calculó el tamaño de efecto $(T E)$ d de Cohen, para las comparaciones intra-grupo y entre-grupos. Por último, se realizó un análisis de potencia.
\end{abstract}

\title{
RESULTADOS
}

Los datos del desempeño motor y de la actividad eléctrica muscular cumplieron con los supuestos de normalidad y homogeneidad $(p>$.05). En la tabla 1 se muestran los valores promedio y desviación estándar para las variables de DM y AEM para cada uno de los grupos y mediciones.

En ANOVA de 2 vías para la variable de desempeño motor (tabla 2) no indicó una interacción significativa, además no se encontró diferencias significativas para el factor grupo. Si se encontró una diferencia significativa en el factor de mediciones. Los análisis post-hoc respectivos determinaron que las tres mediciones son diferentes entre sí, presentando una disminución del tiempo de ejecución en todos los participantes a lo largo del estudio (figura 3 ).

En ANOVA de 2 vías para la variable de la actividad eléctrica muscular (tabla 2) no indicó una interacción significativa, además no se encontró diferencias significativas para el factor grupo, ni medición (figura 4). 
Tabla 1.

Valores de estadística descriptiva para el desempeño motor y actividad eléctrica muscular

\begin{tabular}{|c|c|c|c|c|c|c|c|c|}
\hline \multirow{2}{*}{ Variable } & \multirow{2}{*}{ Grupo } & \multirow{2}{*}{$\mathrm{n}$} & \multicolumn{2}{|c|}{ Pretest } & \multicolumn{2}{|c|}{ Adquisición } & \multicolumn{2}{|c|}{ Retención } \\
\hline & & & $M$ & $D E$ & $M$ & $D E$ & $M$ & $D E$ \\
\hline \multirow[t]{4}{*}{ DM } & PM & 9 & 10.43 & 2.06 & 9.16 & 1.99 & 7.86 & 1.18 \\
\hline & PF & 9 & 10.59 & 2.34 & 8.62 & 1.49 & 7.77 & 0.82 \\
\hline & PC & 6 & 10.82 & 1.52 & 7.59 & 1.73 & 7.04 & 0.93 \\
\hline & Total & 24 & 10.59 & 1.98 & 8.57 & 1.79 & 7.62 & 1.01 \\
\hline \multirow[t]{4}{*}{ AEM } & PM & 9 & 315.72 & 181.25 & 351.66 & 174.86 & NA & NA \\
\hline & PF & 9 & 253.08 & 141.89 & 271.27 & 171.04 & NA & NA \\
\hline & PC & 9 & 313.88 & 140.65 & 356.11 & 140.35 & NA & NA \\
\hline & Total & 27 & 294.23 & 152.54 & 326.35 & 161.40 & & \\
\hline
\end{tabular}

Nota: NA = no aplica. Las unidades de la prueba de desempeño son segundos, las unidades de la AEM son microvoltios. Fuente: elaboración propia.

Tabla 2.

Resultados de ANOVA para desempeño motor y actividad eléctrica muscular

\begin{tabular}{lrrrrrr}
\hline & \multicolumn{3}{c}{ DM } & \multicolumn{4}{c}{ AEM } \\
\cline { 2 - 7 } Factor & \multicolumn{1}{c}{$F$} & $p$ & $\begin{array}{c}\text { Eta parcial } \\
\text { cuadrado }\end{array}$ & $F$ & $p$ & $\begin{array}{r}\text { Eta parcial } \\
\text { cuadrado }\end{array}$ \\
\hline Grupo & 0.56 & .56 & .053 & 0.67 & .52 & .053 \\
Medición & 28.02 & $<.001$ & .572 & 3.07 & .09 & .114 \\
Grupo x Medición & 0.89 & .47 & .078 & 0.15 & .85 & .013
\end{tabular}

Fuente: elaboración propia. 


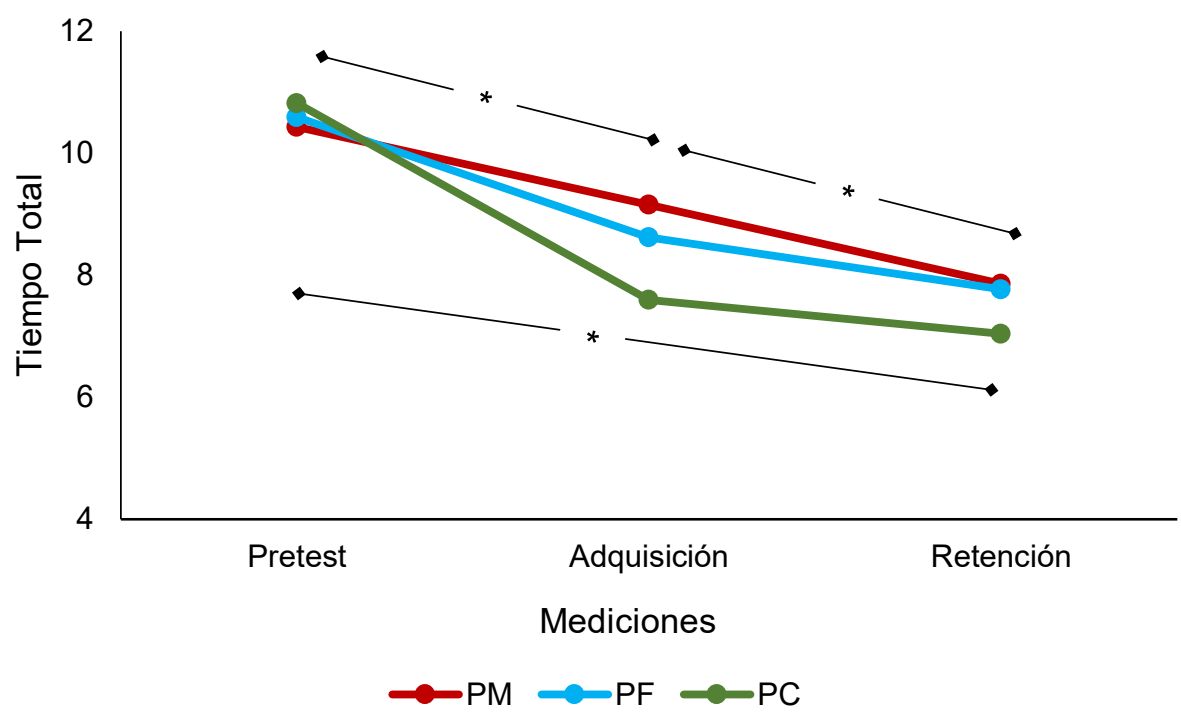

Figura 3. Desempeño motor de los participantes de la destreza de dribbling, para cada medición según el grupo de práctica. *diferencia significativa entre la medición pretest y adquisición, entre la adquisición y la retención, y entre el pretest y la retención $(p<0.05)$.Fuente: elaboración propia.

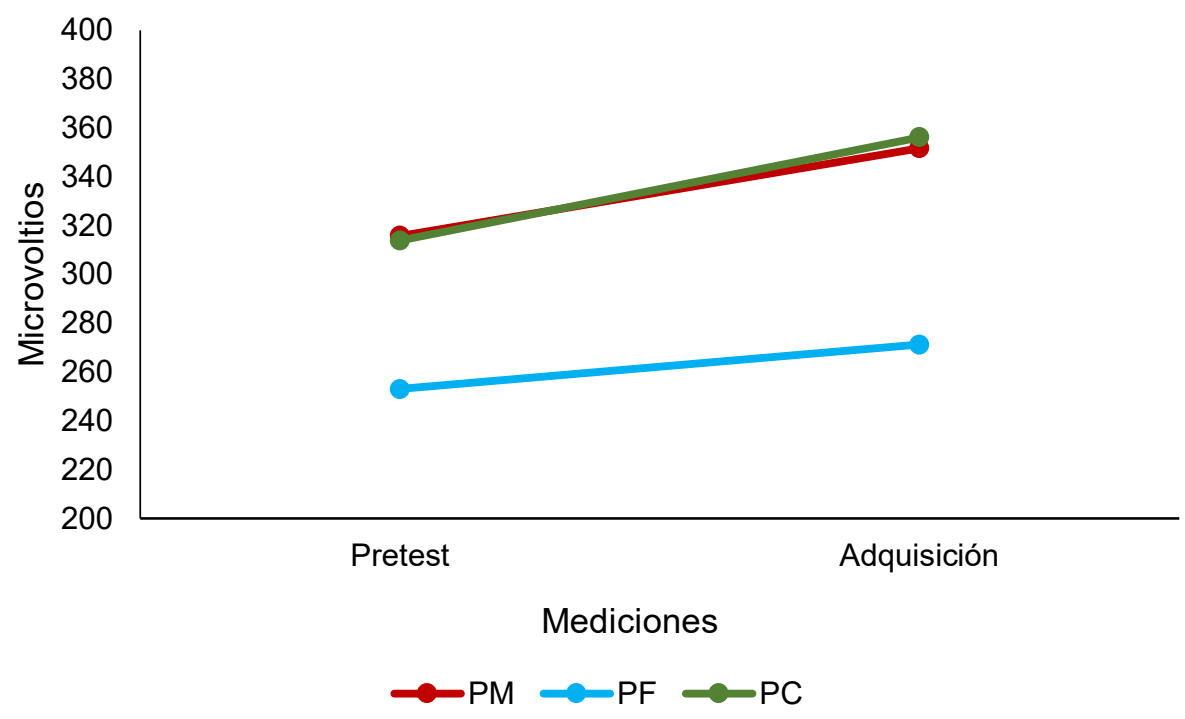

Figura 4. Actividad eléctrica muscular de los participantes de la destreza de dribbling, para cada medición según el grupo de práctica. Fuente: elaboración propia.

Además, en la tabla 3 se presentan los TE calculados intra-grupo, para cada grupo y total de la muestra, entre mediciones, se calculó un TE entre la medición pretest y adquisición, entre la 
medición de adquisición y retención, y entre la medición pretest y retención, los TE negativos implica una disminución en el tiempo, por lo que indica un mejor desempeño. En la tabla 4 se presentan las comparaciones por pares entre-grupos. Por último, dado que el tamaño de muestra puede ser considerado pequeño, y afectar la potencia del estudio, que es la capacidad de detectar un efecto significativo; se calculó a posteriori la potencia del estudio, sugiriendo un valor de $60 \%$, lo que limita detectar un efecto en el $60 \%$ de las veces (Thomas, Nelson y Silverman, 2005).

Tabla 3.

Tamaños de Efecto de cambios obtenidos a lo largo del estudio

\begin{tabular}{lccc}
\hline TE & $\begin{array}{c}\text { Pretest }- \\
\text { adquisición }\end{array}$ & $\begin{array}{c}\text { Adquisición - } \\
\text { retención }\end{array}$ & $\begin{array}{c}\text { Pretest - } \\
\text { retención }\end{array}$ \\
\hline PM & -0.62 & -0.65 & -2.18 \\
PF & -0.84 & -0.57 & -3.44 \\
PC & -2.13 & -0.32 & -4.06 \\
Total & -1.02 & -0.53 & -2.94 \\
\hline
\end{tabular}

Nota: TE = tamaño de efecto. Fuente: elaboración propia.

Tabla 4.

Tamaños de efecto al realizar comparaciones pareadas entre los tipos de práctica

\begin{tabular}{ccc}
\hline TE & Adquisición & Retención \\
\hline $\mathrm{PM}-\mathrm{PF}^{\circ}$ & 0.36 & 0.11 \\
$\mathrm{PC}-\mathrm{PF}^{+}$ & -0.69 & -0.89 \\
$\mathrm{PM}-\mathrm{PC}^{\Phi}$ & 0.90 & 0.88
\end{tabular}

Nota: TE = tamaño de efecto. ${ }^{\circ}$ El PF presentó mayor efecto que el PM. ${ }^{+} E I$ PC presentó mayor efecto que el PF. ${ }^{\oplus}$ PC presentó mayor efecto que el PM. Fuente: elaboración propia.

\section{DISCUSIÓN}

El objetivo del presente estudio fue analizar la efectividad de la PM, PF y su combinación en el desempeño de la destreza de dribbling de hockey de salón y la actividad eléctrica del músculo braquiorradial del antebrazo dominante en estudiantes universitarios novatos. Los resultados del presente estudio apoyan la primera hipótesis de forma parcial, ya que sugieren 
que los tres tipos de práctica favorecen el desempeño y aprendizaje motor de la destreza en cuestión, sin embargo, no hubo cambios en la AEM. No hay evidencia para apoyar la segunda hipótesis. No obstante, los TE calculados indican que, en la adquisición y retención, la PF fue mejor que la PM y la PC fue mejor que la PM y la PF.

Los resultados del presente estudio son consistentes, de forma parcial, con investigaciones previas que han encontrado un efecto positivo de la PF y PC, no así de la PM (Azimkhani et al., 2013; Gomes et al., 2014). Sin embargo, el beneficio de la PM encontrado en este estudio, es congruente con los resultados de estudios previos (Feltz y Landers, 1983; Hegazy et al., 2015). Además, contrario a los resultados del presente estudio, Navarro et al. (2002) encontraron que la PC tiende a favorecer más el desempeño en comparación con PF.

El aprendizaje de una destreza requiere de varios procesos cognitivos y físicos, tales como el procesamiento de estímulos sensoriales, la toma de decisiones, la dirección del movimiento además de la fuerza con la que se debe ejecutar (Ruffino, Papaxanthis y Lebon, 2017), estos procesos se pueden consolidar por distintos tipos de práctica (PF, PM, PC) y generar diferentes ganancias en términos del rendimiento observado y de las modificaciones corticales que se están llevando a cabo (Bassolino, Campanella, Bove, Pozzo y Fadiga 2014; Ruffino et al., 2017). El beneficio encontrado en la adquisición y retención de la destreza, se puede deber, en parte, a que la repetición constante de la destreza durante los tres tipos de práctica, fue un factor relevante para consolidar estos procesos, ya que conllevan a que se realice de manera más rápida, precisa y con un menor esfuerzo cognitivo, actuando directamente sobre la corteza motora primaria, la cual es una región importante en la planificación y ejecución de las acciones (Avanzino et al., 2015).

Existen varios factores (habilidad de los participantes, cantidad de intentos, características de la destreza) que pudieron influir en los resultados del presente estudio, y que los tres tipos de práctica presentaran beneficios similares entre sí en el desempeño de la destreza. En estudios previos se ha sugerido que la PM por sí sola no es beneficiosa en participantes novatos, ya que requieren de la experiencia física previa para realizar una PM eficiente (Gomes et al., 2014); aunque, esto no ha sido apoyado por evidencias metaanalíticas (Feltz y Landers, 1983). Es posible que, si bien los participantes no fueran expertos en la destreza, ni la hayan realizado recientemente, asociaran la ejecución del dribbling a otro deporte y, además, es posible que la realización del pretest, el cual incluyó dos intentos de la destreza, haya sido experiencia suficiente, para permitir una correcta imaginación del movimiento, facilitando el almacenamiento y procesamiento de la información y así mejorar el desempeño en las próximas ejecuciones. 
Por otro lado, en estudios similares se han encontrado beneficios de práctica física y combinada con pocos intentos, no así para la PM (Gomes et al., 2014). Frank et al. (2014) indican que la PM promueve una adaptación cognitiva durante el aprendizaje motor llevando a que los patrones motores aprendidos sean mejor almacenados y faciliten su recuperación en futuras prácticas con rendimientos más precisos. Es posible, en este caso, que la cantidad de intentos realizados durante la práctica fue suficiente para que todos los grupos se beneficiaran.

Otra posible explicación de los resultados son las características de la tarea que está siendo evaluada, ya que aparte de ser nueva para los participantes requería de mucha coordinación óculo-manual, debido a que esta implica tener control en el manejo del palo de hockey y de la pelota. Las destrezas con más componentes cognitivos presentan mayor beneficio de la PM, que las destrezas con componentes motores (Feltz y Landers, 1983). Es posible que en esta destreza se presenten más componentes cognitivos que motrices, por lo que la destreza está siendo más favorecida con la PM, lo que puede estar ocasionando que no haya diferencias entre los tipos de práctica realizados.

Los beneficios de la PM se deben principalmente, a la activación cerebral que se presenta en regiones relevantes de la corteza cerebral, para el proceso de programación e iniciación del aprendizaje motor, durante la actividad de imaginar la ejecución de la destreza (Jackson et al., 2003; Lotze et al., 1999; Luft et al., 1998; Stecklow et al., 2010). Adicional a la activación cerebral, también se ha encontrado activación eléctrica en el músculo en la PM (Guillot et al., 2007; Sánchez y Lejeune, 1999). Por otro lado, es común que un movimiento eficiente presente un menor gasto energético, representado en una disminución de la AEM (Brueckner, Göpfert, Kiss y Muehlbauer, 2019). Sin embargo, en el presente no se encontró cambios en la AEM del braquiorradial del antebrazo dominante, después de realizar la práctica.

Los entrenadores pueden alternar el uso de PF con PM durante las sesiones de trabajo, ya que se puede reducir la fatiga de los participantes cuando la sesión presenta una carga mayor de trabajo y así reducir el riesgo de lesión o dado el caso recuperarse de una lesión. Por ejemplo, Rozand et al. (2014) encontraron que la PM no causa fatiga ni cambios en la fuerza, por el contrario, encontraron que la PF y la PC aumenta la fatiga y también reduce los niveles de fuerza. Por lo que se podría utilizar la PM para aumentar el volumen de entrenamiento sin causar ninguna lesión por sobrecarga de trabajo (Paravlic et al. 2018). Además, si la persona ya presenta una lesión, se puede utilizar este método para atenuar la disminución del desempeño, ya que se ha encontrado que la PM es beneficiosa en el proceso de recuperación durante inmovilizaciones de muñeca (Slimani, Tod, Chaabene, Miarka y Chamari, 2016). Además, en las clases de Educación Física, el profesor puede utilizar la PM como estrategia para el trabajo por 
estaciones, cuando se tiene poco espacio y material en clases con muchos estudiantes. Lo anterior sin poner el riesgo el aprendizaje de la destreza.

No obstante, los resultados deben interpretarse con cautela debido a limitaciones presentes en el estudio. Algunas limitaciones encontradas fue no poder evaluar la AEM durante la práctica realizada, y así obtener un mejor control sobre las señales musculares. Además, la capacidad de imaginación de las personas participantes del grupo de PM y PC también debe ser considerada para futuros estudios, ya que personas con una mejor capacidad tienen representaciones mentales más vívidas y reales que quienes puntúan más bajo, lo que puede influir en los beneficios de la PM. Similar a la anterior, otra limitación, puede haber sido la de no realizar mediciones de cronometría mental —compara el tiempo que tarda haciendo el movimiento de manera real con el imaginado (Dahm y Rieger, 2016) — lo cual hubiera permitido conocer si la percepción del movimiento se interiorizó de manera adecuada, tal y como ha sido demostrada en otros estudios. A su vez, cabe mencionar que otras limitaciones son la ausencia del grupo control y el tamaño de la muestra, esta última siendo afectada aún más por la muerte experimental. De manera complementaria, el análisis de potencia a posteriori sugiere un valor beta de $60 \%$, el cual se encuentra en el límite inferior de los valores recomendados (50\% a $90 \%$ ) por Keppel y Wickens (2004), pero menor al 80\% recomendado en investigaciones en el área del movimiento humano (Thomas et al., 2005). Este valor beta se puede establecer según criterios del investigador, bajo parámetros recomendados, en este caso el $60 \%$ fue suficiente para detectar diferencias en el factor de medición; no obstante, no fue suficiente para detectar diferencias en la interacción, entre los grupos y las mediciones; según las características de la destreza y la cantidad de intentos del presente estudio. El tamaño de muestra, el valor alfa y el efecto del tratamiento son factores que interactúan en la potencia (Keppel y Wickens, 2004); por lo que cabe destacar que en el presente estudio, se buscó un equilibrio fortaleciendo la precisión en la medición al utilizar fotoceldas para la evaluación del desempeño, la homogeneidad de la muestra y el control de las actividades durante la realización de la práctica.

El presente estudio se enfocó en el efecto de la PM, PF y PC en el desempeño y el aprendizaje de una destreza nueva, relacionada al hockey de salón, en estudiantes universitarios sanos, sin conocimiento o experiencia en la destreza. Los tres tipos de práctica favorecieron la adquisición y retención en el desempeño, por lo que se propone el uso de PM y PC, como métodos alternativos o complementarios a la tradicional PF, en contextos de destrezas y poblaciones similares a los del presente estudio, brindando diversidad en las actividades realizadas durante la clase de Educación Física, sin generar más trabajo o fatiga, ya que la PM se podría aplicar en los periodos de descanso o en personas con lesiones. 


\section{AGRADECIMIENTOS}

Se agradece al Centro de Investigación en Ciencias del Movimiento Humano (CIMOHU) de la Universidad de Costa Rica, por el apoyo brindado para la ejecución de este proyecto y al señor Evencio Elizondo Vargas por la colaboración durante la recolección de los datos.

\section{REFERENCIAS}

Avanzino, L., Gueugneau, N., Bisio, A., Ruggeri, P., Papaxanthis, C., y Bove, M. (2015). Motor cortical plasticity induced by motor learning through mental practice. Frontiers in behavioral neuroscience, 9, 105. doi: https://doi.org/10.3389/fnbeh.2015.00105

Azimkhani, A., Abbasian, S., Ashkani, A., y Gürsoy, R. (2013). The combination of mental and physical practices is better for instruction of a new skill. Nigde University Journal of Physical Education And Sport Sciences, 7(2). Recuperado de https://pdfs.semanticscholar.org/c040/35395002cc981df71f06434703ba3f35ccc9.pdf

Bassolino, M., Campanella, M., Bove, M., Pozzo, T., y Fadiga, L. (2014). Training the motor cortex by observing the actions of others during immobilization. Cerebral Cortex, 24(12), 3268-3276. doi: https://doi.org/10.1093/cercor/bht190

Brueckner, D., Göpfert, B., Kiss, R., y Muehlbauer, T. (2019). Effects of motor practice on learning a dynamic balance task in healthy young adults: A wavelet-based time-frequency analysis. Gait \& posture, 70, 264-269. doi: https://doi.org/10.1016/j.gaitpost.2019.03.019

Crews, R. T., y Kamen, G. (2006). Motor-evoked potentials following imagery and limb disuse. International journal of neuroscience, 116(5), 639-651. doi: https://doi.org/10.1080/00207450600592198

Dahm, S. F., y Rieger, M. (2016). Is there symmetry in motor imagery? Exploring different versions of the mental chronometry paradigm. Attention, Perception, \& Psychophysics, 78(6), 1794-1805. doi: https://doi.org/10.3758/s13414-016-1112-9

Feltz, D. L., y Landers, D. M. (1983). The effects of mental practice on motor skill learning and performance: A meta-analysis. Journal of Sport and Excercise Psychology, 5(1), 25-57. doi: https://doi.org/10.1123/jsp.5.1.25

Frenkel, M. O., Herzig, D. S., Gebhard, F., Mayer, J., Becker, C., y Einsiedel, T. (2014). Mental practice maintains range of motion despite forearm immobilization: A pilot study in healthy 
persons. Journal of rehabilitation medicine, 46(3), 225-232. doi: https://doi.org/10.2340/16501977-1263

Gomes, T. V. B., Ugrinowitsch, H., Marinho, N., Shea, J. B., Raisbeck, L. D., y Benda, R. N. (2014). Effects of Mental Practice in Novice Learners in a Serial Positioning Skill Acquisition. Perceptual and Motor Skills, 119(2), 397-414. doi: https://doi.org/10.2466/23.PMS.119c20z4

Guillot, A., Collet, C., Nguyen, V., Malouin, F., Richards, C., y Doyon, J. (2009). Brain activity during visual versus kinesthetic imagery: An fMRI study. Human Brain Mapping, 30(7), 2157-2172. doi: https://doi.org/10.1002/hbm.20658

Guillot, A., Lebon, F., Rouffet, D., Champely, S., Doyon, J., y Collet, C. (2007). Muscular responses during motor imagery as a function of muscle contraction types. International Journal of Psychophysiology, 66(1), 18-27. doi: https://doi.org/10.1016/j.ijpsycho.2007.05.009

Hegazy, K., Sherif, A.M., y Houta, S.S. (2015). The effect of mental training on motor performance of tennis and field hockey strokes in novice players. Advances in Physical Education, 5(2), 77-83. doi: https://doi.org/10.4236/ape.2015.52010

Jackson, P. L., Lafleur, M. F., Malouin, F., Richards, C. L., y Doyon, J. (2003). Functional cerebral reorganization following motor sequence learning through mental practice with motor imagery. Neuroimage, 20(2), 1171-1180. doi: https://doi.org/10.1016/s1053-8119(03)00369-0

Keppel, G., y Wickens, T. D. (2004). Design and Analysis: A Researcher's Handbook. Prentice Hall. Recuperado de https://books.google.com/books?id=SOckAQAAIAAJ

Konrad, P. (2005). The ABC of EMG: A practical introduction to kinesiological electromyography. Recuperado de https://www.researchgate.net/publication/270895853 The abc of emg

Liu, H., Song, L.P., y Zhang, T. (2014). Mental practice combined with physical practice to enhance hand recovery in stroke patients. Behavioural neurology, 2014. doi: https://doi.org/10.1155/2014/876416

Lotze, M., Montoya, P., Erb, M., Hülsmann, E., Flor, H., Klose, U., Birbaumer, N., y Grodd, W. (1999). Activation of cortical and cerebellar motor areas during executed and imagined hand movements: An fMRI study. Journal of cognitive neuroscience, 11(5), 491-501. doi: https://doi.org/10.1162/089892999563553

Luft, A. R., Skalej, M., Stefanou, A., Klose, U., y Voigt, K. (1998). Comparing motion-and imagery-related activation in the human cerebellum: A functional MRI study. Human brain 
mapping,

$6(2)$

105-113.

doi:

https://doi.org/10.1002/(sici)1097-0193(1998)6:2\%3C105::aid-hbm3\%3E3.0.co;2-7

Magill, R. A., y Anderson, D. (2013). Motor Learning and Control: Concepts and Applications (10 ${ }^{\text {th }}$ ed.). McGraw-Hill Higher Education. Recuperado de https://www.amazon.com/-/es/Richard-Magill/dp/0078022673

Matsuda, T., Watanabe, S., Kuruma, H., Murakami, Y., Watanabe, R., Senoo, A., y Yonemoto, K. (2011). Neural Correlates of Chopsticks Exercise for the Non-Dominant Hand; Comparison Among the Movement, Images and Imitations. Rigakuryoho Kagaku, 26(1), 117-122. doi: https://doi.org/10.1589/rika.26.117

Miller, K.J., Schalk, G., Fetz, E.E., den Nijs, M., Ojemann, J.G., y Rao, R.P. (2010). Cortical activity during motor execution, motor imagery, and imagery-based online feedback. Proceedings of the National Academy of Sciences, 107(9), 4430-4435. doi: https://doi.org/10.1073/pnas.0913697107

Navarro, I., Araya-Vargas, G. A., y Salazar, W. (2002). Entrenamiento mental en karatecas: Efecto del tiempo de imaginación de una kata sobre el nivel de ejecución. Pensar en Movimiento: Revista de Ciencias del Ejercicio y la Salud, 2(1), 55-60. doi: https://doi.org/10.15517/pensarmov.v2i1.435

Olusoga, P., Maynard, I., Butt, J., y Hays, K. (2014). Coaching under pressure: Mental skills training for sports coaches. Sport \& Exercise Psychology Review, 10(3), 31-44. Recuperado

de https://www.researchgate.net/publication/272794376 Coaching under pressure Mental skills training for sports coaches

Pangrazi, R. P., y Beighle, A. (2019). Dynamic physical education for elementary school children (19 ed. $^{\text {th }}$ edllinois: Human Kinetics Publishers. Recuperado de https://www.amazon.com/Dynamic-Physical-Education-Elementary-Children/dp/14925922 $\underline{85}$

Paravlic, A. H., Slimani, M., Tod, D., Marusic, U., Milanovic, Z., y Pisot, R. (2018). Effects and dose-response relationships of motor imagery practice on strength development in healthy adult populations: a systematic review and meta-analysis. Sports Medicine, 48(5), 1165-1187. doi: https://doi.org/10.1007/s40279-018-0874-8

Reaz, M. B. I., Hussain, M. S., y Mohd-Yasin, F. (2006). Techniques of EMG signal analysis: Detection, processing, classification and applications (Correction). Biological procedures online, 8(1), 163. doi: https://doi.org/10.1251/bpo124 
Rozand, V., Lebon, F., Papaxanthis, C., y Lepers, R. (2014). Does a mental training session induce neuromuscular fatigue? Medicine \& Science in Sports \& Exercise, 46(10), $1981-$ 1989. doi: https://doi.org/10.1249/mss.0000000000000327

Ruffino, C., Papaxanthis, C., y Lebon, F. (2017). Neural plasticity during motor learning with motor imagery practice: Review and perspectives. Neuroscience, 341, 61-78. doi: 10.1016/j.neuroscience.2016.11.023

Sánchez, X., y Lejeune, M. (1999). Práctica mental y deporte: En qué sabemos después de casi un siglo de investigaciónn. Revista de psicología del deporte, 8(1), 21-37. Recuperado de http://www.rpd-online.com/article/download/89/89

Shea, C.H., y Wright, D.L. (1997). An Introduction to Human Movement: The Sciences of Physical Education. Allyn and Bacon. Recuperado de https://www.amazon.com/-/es/Charles-H-Shea/dp/0137951132

Slimani, M., Tod, D., Chaabene, H., Miarka, B., y Chamari, K. (2016). Effects of mental imagery on muscular strength in healthy and patient participants: A systematic review. Journal of $\begin{array}{llll}\text { sports } \quad \text { science } \quad \text { medicine, } & \text { 434-450. }\end{array}$ https://www.ncbi.nlm.nih.gov/pmc/articles/PMC4974856/pdf/jssm-15-434.pdf

Stecklow, M.V., Infantosi, A.F.C., y Cagy, M. (2010). EEG changes during sequences of visual and kinesthetic motor imagery. Arquivos de neuro-psiquiatria, 68(4), 556-561. doi: https://doi.org/10.1590/s0004-282x2010000400015

Stegeman, D., y Hermens, H. (2007). Standards for surface electromyography: The European project Surface EMG for non-invasive assessment of muscles (SENIAM). Enschede: Roessingh Research and Development, 108-12. Recuperado de http://citeseerx.ist.psu.edu/viewdoc/download?doi=10.1.1.623.2040\&rep=rep1\&type=pdf

Thomas, J.R., Nelson, J.K., y Silverman, S. (2005). Research Methods in Physical Activity (5 ed.). Human Kinetics.

Vodičar, J., Kovač, E., y Tušak, M. (2012). Effectiveness of athletes' pre-competition mental preparation. Kinesiologia Slovenica, 18(1), 22-37. Recuperado de https://www.usfx.bo/nueva/vicerrectorado/citas/SALUD 10/Fisioterapia y Kinesiologia/56 .pdf

Wriessnegger, S.C., Steyrl, D., Koschutnig, K., y Müller-Putz, G.R. (2014). Short time sports exercise boosts motor imagery patterns: Implications of mental practice in rehabilitation programs. Frontiers in human neuroscience, 8. doi: https://doi.org/10.3389/fnhum.2014.00469 
Zapała, D., Zabielska-Mendyk, E., Cudo, A., Krzysztofiak, A., Augustynowicz, P., y Francuz, P. (2014). Short-Term Kinesthetic Training for Sensorimotor Rhythms: Effects in Experts and Amateurs. Journal of Motor Behavior, 47(4), 312-318. doi: https://doi.org/10.1080/00222895.2014.982067 